\title{
El comercio exterior de Malasia en el marco de la apertura económica
}

\section{Introducción}

El objetivo de este trabajo es mostrar un panorama general de los acontecimientos más notables en la economía de Malasia, así como las repercusiones que en ella ha tenido la apertura comercial. El trabajo se compone de tres apartados: el primero se refiere a la evolución de la economía de Malasia, donde se hace alusión a los acontecimientos económicos notables que la llevaron a dar giros importantes en la orientación de la economía y a las políticas comerciales empleadas; el segundo apartado habla de la importancia de la Inversión Extranjera Directa (IED) para los planes estratégicos seguidos por Malasia; el tercero analiza los acontecimientos relacionados con el comercio exterior, donde se examina la composición del comercio y las barreras arancelarias y no arancelarias impuestas por el país, sus principales socios comerciales y la importancia de los acuerdos o tratados suscritos para su economía. Finalmente, se presentan algunas reflexiones de carácter general sobre el proceso de apertura económica de Malasia.

\section{La apertura de la economía}

Desde fines de la época colonial (1957), Malasia experimentó un rápido crecimiento económico y orientó sus actividades productivas hacia los mercados

Profesora investigadora del Departamento de Estudios del Pacífico de la Universidad de Guadalajara. 
de exportación. La composición del comercio fue principalmente de productos agrícolas, entre los que destacan: aceites vegetales, caucho natural, estaño, petróleo y gas natural. Las altas tasas de crecimiento atrajeron inmigrantes de países vecinos (Indonesia, Tailandia y Filipinas) que poseían abundante mano de obra no calificada. El panorama era prometedor para los inmigrantes y muchos de ellos llegaron para quedarse, lo que abarató los costos para los grandes productores de los cultivos que abastecieron el consumo interno y de exportación, en detrimento de los pobladores locales.

A principios de los años ochenta, el gobierno de Malasia planeó transformar su base económica, enfocarse a productos de alta tecnología con mayor valor añadido. En 1987 el gobierno malayo intensificó la participación gubernamental en el desarrollo urbano e industrial. Se trabajó en un plan económico de reforzamiento de la industria, específicamente en la manufactura de productos eléctricos y electrónicos. Para tal efecto, reforzaron acciones para atraer inversión extranjera directa (IED) a Malasia; así llegaron empresas de Estados Unidos, Europa y Japón aprovechando las facilidades otorgadas a la inversión y la existencia de mano de obra de bajo costo.

Esta situación trajo consigo inmigración hacia las zonas urbana en busca de una vida mejor, dadas las precarias condiciones en las regiones rurales. Sin embargo, el gobierno de Malasia no tuvo la precaución de incluir en su plan económico programas educativos que prepararan el capital humano para las nuevas tecnologías que se instalarían en Malasia, lo que recrudeció el problema de la inmigración y obligó al país a contratar mano de obra extranjera dada la creciente demanda laboral como consecuencia de las altas tasas de crecimiento económico.

Durante la década 1980-1990, el crecimiento del producto interno bruto (PIB) real fue de 5.2\% en promedio anual, y en el periodo 1990-1995 se observó un marcado aceleramiento de $8.7 \% .{ }^{1}$ De esta forma, la industria manufacturera ganaba terreno al resto de los sectores; de 1991-1995 representó en promedio anual $77.4 \%$ del total exportado, después de haber tenido en el quinquenio anterior tan solo $19 \%^{2}$ del sector, por lo que a este acontecimiento se le nombró "el milagro industrial de Malasia".

1. Felker Grez y K. S. Jomo, Technology, Competitiveness and the State, Malasia's industrial technology policies, Routledge Taylor \& Francus Group, Londres y New York, 1999, p. 2.

2. İdem. 
En 1997-1998 se interrumpió el crecimiento económico debido a la crisis asiática financiera; sin embargo, su recuperación fue más rápida que en el resto de los países involucrados. Se cree que el mérito fue haber salido por sus propios medios, sin atender las recomendaciones del Fondo Monetario Internacional. Este favorable desempeño económico de Malasia atrajo las miradas internacionales, lo que dio lugar a que Estados Unidos y la Unión Europea, en 1998 y 1999 respectivamente, le retiraran los beneficios del Sistema Generalizado de Preferencias (SGP), ${ }^{3}$ porque consideraron que Malasia había salido de la condición de país en vías de desarrollo.

No obstante su rápida recuperación de la crisis, el destino le deparaba otro tropiezo: la alta dependencia de las exportaciones de productos manufacturados (eléctricos y electrónicos principalmente), en medio de la desaceleración de la economía mundial en 2001, llevó Malasia a una recesión económica que tiró por la borda "el milagro industrial de Malasia", debido a la disminución de la demanda internacional de productos de alta tecnolo-

La alta dependencia de las exportaciones de productos manufacturados, en medio de la desaceleración de la economía mundial en 2001, llevó Malasia a una recesión económica que tiró por la borda "el milagro industrial de Malasia", gía. El crecimiento del PIB en 2001 fue tan solo de $0.3 \%$ (cuadro 1 ).

Las experiencias vividas por Malasia motivaron al gobierno a planear nuevas políticas que impulsaran los sectores estratégicos de la economía. En 2006 se logró un crecimiento del PIB de 5.9\% (cuadro 1); no obstante, la disparidad en la distribución del ingreso fue cada vez más pronunciada, por lo que se consideró pertinente tomar medidas que llevaran a la economía a

3. El Sistema Generalizado de Preferencias de los Estados Unidos (SGP) se implementó en 1974. Es una herramienta de política comercial unilateral del gobierno de Estados Unidos, cuyo objetivo consiste en promover el crecimiento económico de los países en desarrollo y de los países menos adelantados, beneficiarios del programa. Para el logro de dicho objetivo, se busca incrementar el comercio de estos países mediante la eliminación de los aranceles de importación de Estados Unidos a más de 4,800 productos. Asimismo y sólo para los países menos adelantados, el sistema prevé un trato exento adicional a más de 1,400 productos de ese origen que sean importados por Estados Unidos. 
un crecimiento sólido e incluyente y con una mínima vulnerabilidad ante los factores externos.

De esta manera, surgió el Noveno Plan de Malasia 2006-2010, en el que se asignó un presupuesto a la agricultura de $70 \%$ mayor que en el plan anterior; la prioridad fue impulsar el sector agrícola y el rescate a la pobreza. Sin perder de vista los beneficios de la apertura comercial, Malasia, al igual que Singapur, es una de las economías con mayor grado de apertura económica en el grupo de países que componen la Asociación de Naciones del Sureste Asiático (ASEAN). ${ }^{4}$ Participa en acuerdos regionales como miembro de la región ASEAN y es miembro activo en el APEC, asume compromisos con la OMC de carácter multilateral y ha suscrito un acuerdo de libre comercio con Japón en 2005; en 2006 Malasia y Estados Unidos iniciaron negociaciones con la posibilidad de firmar un Tratado de Libre Comercio; a finales de 2007 Malasia y Chile iniciaron negociaciones para la posible firma de un Tratado de Libre Comercio.

\section{Evolución de la economía de Malasia}

Con el fin de orientar su política económica de manera más adecuada, el gobierno de Malasia asumió el compromiso de pasar de una economía impulsada por la producción agrícola, a otra basada en el conocimiento y la productividad. Además, organizó sus actividades económicas en planes de acción quinquenales y planes de acción globales de periodos más amplios; desde el Primer Plan de Malasia (1966-1970) hasta el Cuarto Plan, todos los esfuerzos gubernamentales en la economía se dedicaron al desarrollo agrícola y rural.

A partir del periodo que abarca el Cuarto Plan de Malasia 1980-1985, surge la idea de que es el momento de realizar cambios radicales en la economía; su prioridad fue atraer IED para impulsar el sector industrial con el fin de lograr una producción con mayor valor agregado y de alta tecnología, al mismo tiempo daba inicio a la privatización de empresas. Por lo que se tuvo la necesidad de establecer la infraestructura necesaria para empresas de alta tecnología, mediante la construcción de naves industriales, carreteras, medios de comunicación y requerimientos básicos en las zonas industriales, entre otras.

4. Los diez países miembros de la ASEAN son Brunei Darussalam, Camboya, Indonesia, República Democrática Popular Laos, Malasia, Myanmar, Filipinas, Singapur, Tailandia y Vietnam. 
En apariencia los programas de desarrollo incluían al resto de los sectores, además del industrial; lo cierto es que el sector agrícola gradualmente fue perdiendo participación en la economía a medida que ganaba el industrial.

Los resultados no se hicieron esperar: el crecimiento del producto interno bruto (PIB) fue de 8.7\% en promedio anual en el periodo 1990-1995, después de haber mantenido un promedio de 5.2 en 1980-1990, como consecuencia del incremento en la producción en la industria manufacturera, en 1980-1985 representaron $8.9 \%$ y en $1990-1995,13.2 \% .^{5}$

Como consecuencia del crecimiento de la producción y la aceleración en el sector manufacturero, las exportaciones totales se vieron favorecidas, destacando el incremento de las manufacturas respecto a las exportaciones totales. En el periodo 1980-1985 el sector manufacturero exportaba en $23.2 \%$, en cambio el sector agrícola 41.2\%; en el periodo 1991-1995 se invierte la importancia del sector agrícola por el manufacturero, al representar $18.7 \%$ y $68.5 \%$ respectivamente, como porcentaje en las exportaciones totales. ${ }^{6}$ Las importaciones manufactureras ya eran altas desde a principios de los años ochenta, Malasia importaba la mayoría de artículos manufactureros que no se fabricaban en el país (cuadro 4).

\section{Cuadro 1}

Malasia: Indicadores económicos, 1995-2006

\begin{tabular}{lcccc}
\hline & Inflación & Tasa de desempleo & Tipo de Cambio promedio anual & PIB Real crecimiento \\
\hline 1995 & & 2.8 & 2.5 & \\
1996 & 3.5 & 2.5 & 2.5 & 10.0 \\
1997 & 2.7 & 2.5 & 2.8 & 7.3 \\
1998 & 5.3 & 3.2 & 3.9 & -7.4 \\
1999 & 2.7 & 3.5 & 3.8 & 6.1 \\
2000 & 1.5 & 3.1 & 3.8 & 8.9 \\
2001 & 1.4 & 3.5 & 3.8 & 0.3 \\
2002 & 1.9 & 3.5 & 3.8 & 4.4 \\
2003 & 1.0 & 3.6 & 3.8 & 5.5 \\
2004 & 1.5 & 3.6 & 3.8 & 7.2 \\
2005 & 2.9 & 3.6 & 3.8 & 5.0 \\
2006 & 3.6 & 3.3 & 3.7 & 5.9 \\
2007 & 2.1 & 3.2 & 3.4 & 6.3 \\
\hline
\end{tabular}

Fuente: International Montary Fund, International Financial Statistics, Yearbook, 2007.

5. Felker Grez y K. S. Jomo, op. cit., p. 1.

6. Ídem. 
La política económica de Malasia siguió el mismo rumbo en los periodos 1995-2000 y 2000-2006 (Séptimo y Octavo Plan de Malasia). El producto interno bruto manufacturero siguió ganándole terreno al sector agrícola. En 2005 y 2006 el sector industrial representó 51.8\% y 49.9\% del PIB respectivamente, mientras que el sector agrícola tan solo representó $8.7 \%$ en ambos años (cuadro 2).

De esta forma Malasia cayó en un alto grado de dependencia en el sector industrial y muy específicamente en los productos eléctricos y electrónicos. Su afán por alcanzar la especialización industrial hizo colapsar el modelo, fue inevitable que en 2001 cayera en una fase cíclica global de la industria electrónica y por lo tanto en una recesión. Además, la Organización de las Naciones Unidas (ONU), en el Informe sobre Desarrollo Humano, señala que los indicadores de educación no van de acuerdo con los avances en la industrialización (6.8 en promedio de estudios de los adultos) y que aún quedan sectores marginados con altos índices de pobreza: 9.3\% de la población vive con dos dólares al día (2’390,000 personas) y 10\% de la población de mayor ingreso gana 22.1 veces más que $10 \%$ de la población más pobre. Éstos son indicadores importantes que dan la pauta para incluir mecanismos que reduzcan los problemas mencionados.

Cuadro 2

Malasia: Producción por sector como porcentaje del PIB, 1990 y $2004-2006$

\begin{tabular}{lcccc}
\hline & 1990 & 2004 & 2005 & 2006 \\
\hline Agricultura & 15.0 & 10.0 & 8.7 & 8.7 \\
Industria & 42.0 & 50.0 & 51.8 & 49.9 \\
Manufactura & 24.0 & 31.0 & $\ldots$. & $\ldots \ldots$ \\
Servicios & 43.0 & 40.0 & 39.6 & 41.3 \\
\hline
\end{tabular}

.... No disponible

Fuente: World Bank, World Development Indicators, varios números.

A pesar de que Malasia es un país rico en recursos naturales, no se le dio el impulso suficiente para aprovecharlos de manera sustentable. Casi durante 20 años quedó estancado el sector primario: no se le dio la atención adecuada y suficiente para explotar sus ventajas comparativas en productos de origen agrícola. 
Apostar todo a la industria manufacturera, a pesar de no haber resuelto primero los problemas estructurales, provocó que se acentuara la problemática que enfrentan los grupos sociales más vulnerables.

Estos señalamientos, más la experiencia vivida por el colapso de la economía por la alta dependencia de unos cuantos productos, originaron un nuevo plan de la economía de Malasia, donde se pretende impulsar sectores estratégicos y está incluido el sector rural.

De esta forma surge el Noveno Plan de Malasia 2006-2010, en el que, a diferencia de los planes anteriores, se plantea impulsar al sector agrícola y aumentar la calificación de la mano de obra. Se espera que para 2010 la creciente disparidad en la distribución del ingreso se haya revertido por lo menos en $50 \%$, y el crecimiento muestre mayor solidez que en experiencias anteriores. Parte de este plan es la inclusión de nuevas fuentes de crecimiento basadas en la agricultura, los servicios y las industrias de transformación de recursos naturales, con el fin de disminuir la concentración de las exportaciones en la industria manufacturera, en los productos eléctricos y electrónicos. Por el contrario, se pretende fortalecer los sectores que no han sido explotados lo suficiente pero que son una importante fuente de ingresos, sin perder de vista lo peligroso de depender de unos cuantos productos.

Para lograr los objetivos del Noveno Plan de Malasia es importante seguir incentivando la IED en los diferentes sectores de la economía, ya que es un ingrediente fundamental para el impulso del crecimiento económico en Malasia.

\section{Inversión extranjera directa en Malasia}

La orientación de la IED en los sectores económicos del país depende del rumbo de la política que se diseñe para la economía. En Malasia durante aproximadamente 20 años (1985-2006) la IED se dirigió en mayor medida a la industria manufacturera específicamente en productos eléctricos y electrónicos. Aunque también participa en la biotecnología, el petróleo, el gas natural y el sector automovilístico, entre otros. En el noveno plan de Malasia en 2006, se previó una mejor distribución sectorial de la IED, con prioridad en sectores estratégicos, que incluyen el sector agrícola, con el fin de ayudar a disminuir la vulnerabilidad ante cualquier desequilibrio económico interno o externo.

En el camino hacia la globalización económica que Malasia ha trazado, aún quedan acciones pendientes; sin embargo, el avance logrado en la creación de infraestructura en el sector industrial deja de manifiesto que al reorientar su 
economía, seguramente redoblará esfuerzos para lograr lo mismo con otros sectores.

La atracción de IED en condiciones de proceso de apertura económica se ha vuelto indispensable para el funcionamiento de la economía de Malasia. La presencia de empresas multinacionales se ha considerado importante para aliviar la falta de empleo, el creciente empleo informal e impulsar las pequeñas y medianas empresas. Todavía no se logra la vinculación entre las multinacionales y las empresas locales.

A pesar de los importantes cambios en la economía de Malasia, no se han registrado modificaciones o adecuaciones a la Ley de Promoción de las Inversiones y la Ley de Coordinación Industrial que datan de 1986 y 1975, respectivamente. Éstas regulan las inversiones extranjeras y nacionales y garantizan que todas las empresas que hacen negocios en Malasia mantengan al menos $30 \%$ de capital bumiputra (malayos y otros pueblos indígenas). Debido al empeño del gobierno por atraer IED a la industria manufacturera, a partir de diciembre de 2003 se permitió que las empresas extranjeras poseyeran $100 \%$ del capital en las nuevas inversiones, así como en la ampliación de las inversiones ya existentes en empresas manufactureras. ${ }^{7}$

Otro factor importante, que va de la mano con la apertura de la economía, es la privatización de empresas en Malasia. Algunas empresas ligadas al sector público tienen una dimensión muy importante; la principal es "Petronas", empresa que dirige todo el desarrollo de los recursos energéticos del país. Las principales empresas públicas, en los sectores de electricidad, telefonía, transporte aéreo y marina mercante, han sido formalmente privatizadas (aunque el sector público mantiene una situación de control en casi todas ellas). "Los activos que posee el gobierno equivalen a más de la mitad del PIB de Malasia. Las empresas más importantes vinculadas a la administración son Petronas (petróleo y gas), Tenegas (electricidad), Malaysian Airlines, Proton (empresas de automóviles) y Telekon Malaysia". ${ }^{8}$

Sin embargo, en la crisis financiera de 1997-1998 quedó claro que algunos sectores aún no estaban preparados para ser privatizados. Por esa razón el gobierno acudió en ayuda de ciertas empresas, como es el caso de las de telecomunicaciones, infraestructura, transporte urbano, acero y fabricación de automóviles. Recuperó el gobierno varias empresas que había privatizado en

7. OMC, Examen de las Políticas Comerciales de Malasia, 2005.

8. Ídem. 
1983 y que dieron señales de no estar preparadas para la privatización, entre las que destacan la compañía aérea nacional Malaysian Airlines System y el sistema de transporte ligero por ferrocarril de Kuala Lumpur (capital de Malasia).

Malasia mantiene lazos muy estrechos y proyectos conjuntos con el grupo de países de la ASEAN. En 1998 firmaron un acuerdo de inversión con el fin de promover la integración económica y difundir atractivamente la región para la inversión. Esto facilitaría la libre circulación de la inversión directa, la tecnología y los profesionales calificados; en las manufacturas, la agricultura, la pesca, la silvicultura y la minería, así como las actividades de servicios relacionadas con esos sectores. Además de trabajar en el proyecto de un mercado único en la región en 2020, en el cual podrán circular libremente las mercancías, los servicios, la mano de obra calificada y el capital.

Sin embargo, en su gran mayoría la IED llega de países fuera de la región ASEAN, a pesar de las facilidades que se dan para su ingreso al país. A partir de 1998 se atenuaron las prescripciones sobre la participación en el capital y desde el 17 de enero de 2003 se permite que pertenezca a extranjeros 100\% del capital de las inversiones en nuevos proyectos y en la ampliación o diversificación de los proyectos de empresas ya existentes, con independencia del nivel de las exportaciones. Se exige una licencia de fabricación a las empresas cuyos recursos propios ascienden a 2.5 millones de ringgits malayos o más, o que al menos tengan 75 empleados a tiempo completo, de conformidad con lo dispuesto en la Ley de Coordinación Industrial.

Según las autoridades de Malasia, el país es puerta de entrada al mercado de la ASEAN y ofrece incentivos, entre ellos el régimen de las empresas pioneras y desgravaciones fiscales a las inversiones, para las empresas extranjeras que recurran a esa entrada por Malasia. Entre los principales inversores extranjeros destacan Estados Unidos, Singapur, Japón y Hong Kong, los cuales también figuran como sus principales socios comerciales.

\section{El comercio exterior de Malasia}

La apertura económica para Malasia ha sido prioritaria en su política económica; sus metas están trazadas tanto en el ámbito regional como en el multilateral. A escala regional espera cumplir las metas de Bogor en el APEC y conformar el mercado único con los países de la ASEAN en 2020. Por medio de la ASEAN participó en negociaciones regionales de libre comercio e inversión con Australia, Nueva Zelanda, China, India, Corea del Sur. En el ámbito 
bilateral firmó un tratado de libre comercio con Japón. Además, firmó un acuerdo marco sobre comercio e inversión con Estados Unidos, y actualmente se encuentran en negociaciones bilaterales para un tratado de libre comercio con ese país. De igual forma, a finales de 2007 inició negociaciones con Chile para el mismo fin.

El primer acuerdo de tipo económico que Malasia firmó en el exterior fue en 1967 con la ASEAN, que incluye las economías de Malasia, Filipinas, Indonesia, Singapur y Tailandia. Aunque no se habló de un acuerdo regional de libre comercio, sí se realizaron acuerdos para lograr un crecimiento económico sostenido, colaboración y cooperación conjunta para dar solución a algunos problemas en la región. En 1984 se integró Brunei, y no fue sino hasta 1992, tres años antes de la integración de Vietnam (1995), cuando las economías de la ASEAN decidieron que era el momento de integrarse y trabajar en pos de una zona de libre comercio: se contempló una reducción gradual de los aranceles en los productos no agrícolas que comercien entre sí, de tal manera que en quince años (a partir de 1993 y hasta finales de 2008) estarán libres de arancel.

No obstante su histórica relación tan estrecha con países de la región, Malasia exportó $26.1 \%$ e importó $25.5 \%$ de su comercio total; el resto del comercio lo realizó con economías fuera de la región de la ASEAN. ${ }^{9}$ Es decir, tres de sus cuatro socios principales se encuentran fuera de la región, con excepción de Singapur, quien se posiciona en 2005 como el segundo socio comercial más importante después de Estados Unidos y el tercero en importaciones, después de Estados Unidos y Japón (cuadro 3).

Para Malasia es importante disminuir las barreras arancelarias y no arancelarias a fin de consolidar los intercambios comerciales; pretende lograrlo con tratados y acuerdos de libre comercio. La importancia que para Malasia tienen Estados Unidos, Singapur, Japón y China, es porque representan cerca de $50 \%$ de sus exportaciones e importaciones (cuadro 3)

Se considera que ha aumentado la competencia en las industrias de $\mathrm{Ma}$ lasia al haberse eliminado los obstáculos internos por la firma de acuerdos de libre comercio bilaterales y regionales, por las negociaciones de la OMC y porque países con bajos salarios, en particular China, debilitan la aventaja competitiva de Malasia en manufacturas de uso intensivo de mano de obra no calificada.

9. ASEAN, Trade Database, 2006. 


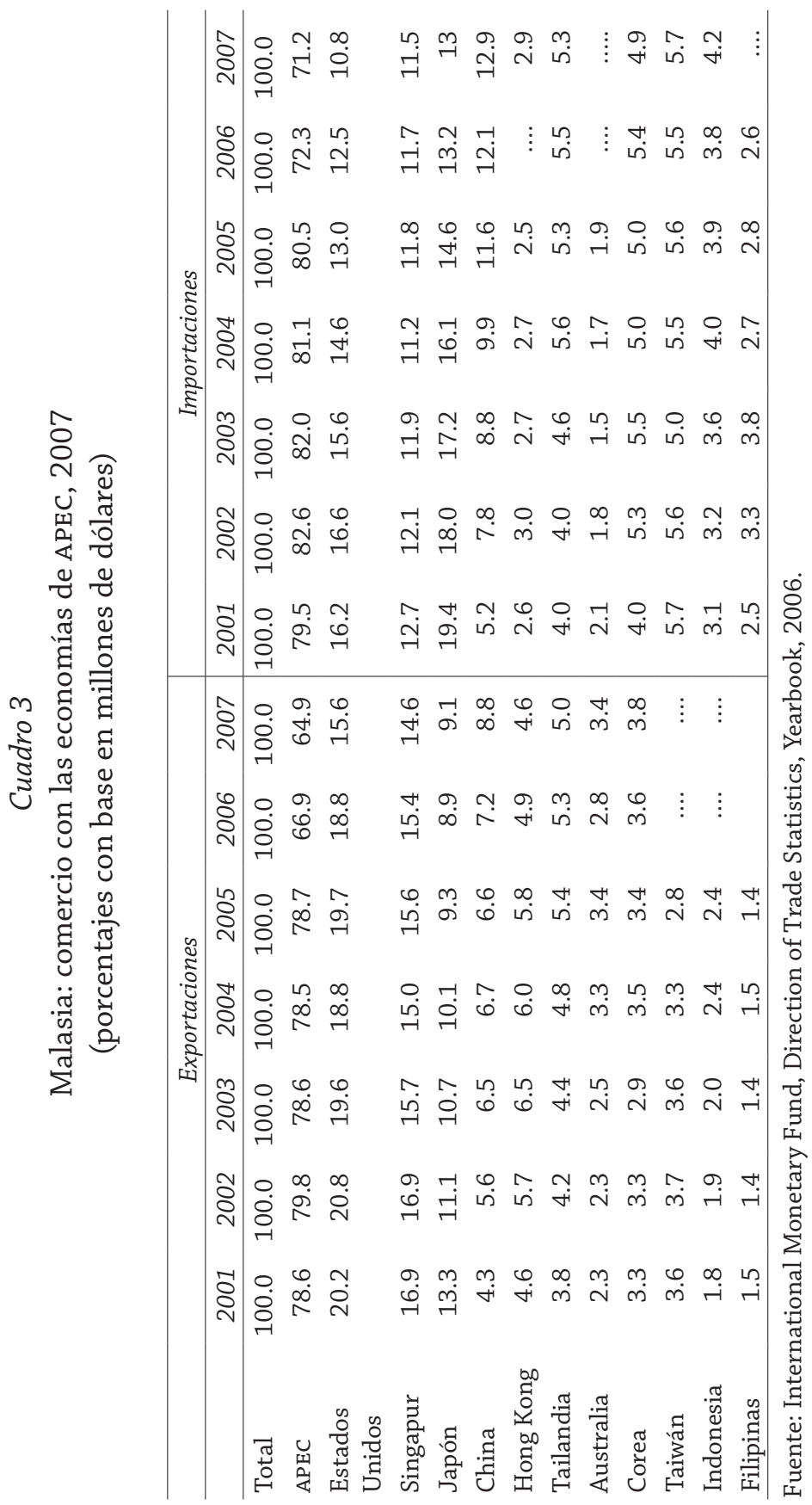


Un cambio importante de las pautas del comercio de Malasia proviene del aumento de la participación de China: en 2004 ocupó el cuarto lugar en importancia, en 2006 y 2007 China se posiciona en tercer lugar como proveedor de importaciones de Malasia sólo después de Estados Unidos y Singapur.

La política relacionada con el comercio tiene como fin general promover y salvaguardar los intereses de Malasia en la esfera del comercio internacional; estimular el desarrollo de las actividades industriales y aumentar el crecimiento económico del país para alcanzar los objetivos del programa Visión 2020. Se considera que la OMC, la Zona de Libre Comercio de la ASEAN y otros acuerdos regionales acentuarán la liberalización del comercio intensificando la competencia en los mercados nacionales e internacionales.

El Octavo Plan de Malasia, que abarca el periodo 2001-2005, consta de estrategias y programas sectoriales encaminados al desarrollo de una economía basada en los conocimientos. El plan tuvo por finalidad sustituir una estrategia de crecimiento basada en insumos intensivos por otra más centrada en los conocimientos, mediante el aumento de la productividad, la reestructuración más rápida de los sectores de manufacturas y servicios, así como la revitalización del sector agropecuario.

Entre iniciativas para promover las exportaciones se incluyen el aumento de la competitividad y la creación de nichos de mercado en determinados sectores como los servicios de enseñanza, turismo, sanidad, transporte y los servicios profesionales. Además de la creación de marcas de prestigio mundial, y la entrada con paso más firme en mercados no tradicionales.

La participación en las negociaciones sobre la agricultura se guía por la necesidad de reducir sustancialmente todas las distorsiones del mercado mundial que suponen una competencia desleal para las exportaciones del país: Malasia desea que se asuman compromisos a fin de eliminar las subvenciones agrícolas y reducir sustancialmente los aranceles elevados.

\section{Barreras arancelarias y no arancelarias en el comercio de Malasia}

A pesar de que la política comercial de Malasia es de apertura, aproximadamente 27\% de las líneas arancelarias en 2004 estuvieron sujetas a licencias de importación y ha recurrido con bastante frecuencia a medidas comerciales especiales (como acciones antidumping). Además, se aplican impuestos de exportación y numerosas disposiciones en materia de licencias a determinadas exportaciones (como la madera) para desalentar su comercio exterior del pro- 
ducto en bruto y fomentar el desarrollo de industrias en fases más avanzadas del proceso de producción. En este caso se presta ayuda a las exportaciones a través de diversas medidas, como las exenciones y devolución de los derechos de importación, desgravaciones fiscales, créditos, seguros y ayuda patrocinada por el gobierno para la promoción y comercialización.

La política comercial de Malasia se usa como un instrumento de política industrial bajo la autoridad del Ministerio de Comercio Internacional e Industria (MITI). A pesar del acuerdo y compromisos asumidos en la Organización Mundial del Comercio (OMC), los aranceles de tipo promedio denominados nación más favorecida (NMF), lograron disminuir de $15.2 \%$ en 1993 hasta 8.1\% en 1997 y que se mantiene en la actualidad.

La protección arancelaria es alta cuando existe producción local (entre $30 \%$ y $50 \%$ ); cuando se va a iniciar la producción en el país, se puede solicitar las tasas de derechos de importación, las cuales van desde $2 \%$ hasta alcanzar más de 100\%, incluso llegan a 145\% ad valorem.

Los casos extremos opuestos en la aplicación o no de barreras arancelarias son los siguientes: por un lado no están sujetos a impuestos las materias primas, los alimentos básicos y los productos farmacéuticos. Por el otro, sectores considerados estratégicos están protegidos por un sistema de licencia restringida de importación, además de la protección arancelaria: es el caso de algunos productos alimentarios básicos, siderurgia, cemento, cerámica, artículos textiles, prendas de vestir, cuero, productos alimenticios, bebidas y automóviles. Este último es muy cuidado en Malasia: además de requerir licencia de importación, está sujeto a un arancel de entre $140 \%$ y $300 \%$, en cambio las autopartes pagan entre $42 \%$ y $80 \%$, por lo que es más conveniente ensamblarlas en el país. Obviamente con los países que mantienen tratados o acuerdos comerciales se establece otro tratamiento, ya que en el acuerdo se estipula el grado de apertura según el caso (de acuerdo con cifras de la OMC).

En el Tratado de Libre Comercio de la ASEAN (AFTA, por sus siglas en inglés) se tomó la decisión de que a partir de 2003, todos los productos dentro del sistema del arancel preferencial efectivo común (CEPT) aportan un arancel de cero a $5 \%$ (los productos agrícolas no procesados se encuentran fuera del acuerdo). Se aplica la reducción o eliminación de aranceles en condiciones de reciprocidad y se aplican prescripciones en materia de contenido regional.

El acuerdo marco de la ASEAN sobre servicios, firmado en 1995, sirve de orientación para liberalizar los servicios más allá de los compromisos asumidos en el marco de la OMC y promueve la cooperación entre los proveedores de 
servicios de la ASEAN. Hasta ahora, la ASEAN ha completado cuatro paquetes de medidas de liberalización de los servicios que abarcan la construcción, las telecomunicaciones, los servicios prestados a las empresas, los servicios financieros, el transporte aéreo y marítimo y el turismo. Dichas medidas incluyen acceso preferencial para los demás estados miembros de la ASEAN, por lo que se refiere al establecimiento de empresas de servicios al empleo de profesionales. Con arreglo a un enfoque de integración prioritario, está previsto liberalizar el turismo, la atención sanitaria y los viajes por avión, a más tardar en 2010. Los países de la ASEAN están trabajando para ampliar las negociaciones, a fin de abarcar todo los sectores y modos de suministro.

En mayo de 2005 Japón y Malasia lograron un acuerdo inicial con el que se suprimirá la mayor parte de los aranceles entre ambos países para 2015. Se trata para Malasia de su primer acuerdo bilateral de libre comercio. En diciembre de 2004, Malasia y la India acordaron concertar un Acuerdo Amplio de Cooperación Económica, que incluyera la posibilidad de establecer un acuerdo de libre comercio. Se estableció un grupo de estudios conjuntos para determinar, entre otras cosas, la manera en que ambos países puedan aumentar el grado de cooperación económica más allá del comercio tradicional de productos agrícolas y, en particular, con la finalidad de elaborar un marco de política a fin de ampliar el comercio de mercancías y servicios y las inversiones (incluida la viabilidad de un acuerdo de libre comercio para las mercancías); ampliar la cooperación económica en esferas de interés mutuo, como la tecnología de la información y las telecomunicaciones, la biotecnología, los productos farmacéuticos, la atención sanitaria, la enseñanza y el turismo, y propiciar la corriente transfronteriza de inversión.

En junio de 2004 Malasia firmó el acuerdo relativo al sistema de comercio preferencial bajo los auspicios de la Organización de la Conferencia Islámica, con los siguientes países: Bangladesh, Camerún, Egipto, Guinea, Irán, Jordania, El Líbano, Pakistán, Senegal, Túnez, Turquía, Uganda y Malasia. Dicho acuerdo prevé concesiones arancelarias preferenciales entre sus miembros para determinadas mercancías. Se están examinando las modalidades de reducción arancelaria. La aplicación de este acuerdo proporcionaría a los exportadores malasios trato arancelario preferencial para determinados productos.

Malasia también es miembro del Acuerdo sobre Aranceles Preferenciales del Grupo de Ocho países en Desarrollo, junto con Bangladesh, Indonesia, Irán, Egipto, Nigeria, Pakistán y Turquía. Dicho acuerdo tiene por objeto otorgar 
concesiones arancelarias preferenciales para determinadas mercancías entre los países participantes.

Malasia ofrece como mínimo el trato NMF acordados en la OMC a todos sus interlocutores comerciales, mientras que otorga preferencias arancelarias a los países de la ASEAN. El promedio del tipo arancelario NMF aplicado es de aproximadamente $8.1 \%$ en 2005, frente a $9.2 \%$ en 2001; el promedio de los tipos consolidados es de $16.2 \%$. Según las autoridades, Malasia seguirá examinando y reduciendo los tipos arancelarios en armonía con su objetivo de conseguir una economía más abierta y liberal, hacer más eficiente la asignación y acentuar la competitividad del entorno para las ramas de producción nacionales.

Malasia no otorga ninguna subvención directa a la exportación. En la Ronda de Doha de negociaciones comerciales, se ha comprometido a eliminar las formas de subvenciones a la exportación otorgadas a todos los productos agropecuarios. Malasia es uno de los mayores exportadores mundiales de semiconductores y se ha trabajado para pasar de la fabricación al análisis y diseño, con el objetivo de mantenerse a la vanguardia.

Las actividades más importantes son la producción de insumos alimenticios y de cultivos para los mercados internacionales, principalmente el aceite de palma, el caucho y la madera; esto con el fin de fomentar el desarrollo de la agricultura.

\section{Composición del comercio de Malasia}

El comercio exterior de Malasia ha manifestado importantes transformaciones en su composición: disminuyeron las exportaciones de productos agrícolas desde finales de los años ochenta y al mismo tiempo se incrementaron las exportaciones manufactureras (cuadro 4).

El plan del gobierno en ese momento fue orientar su inversión al impulso de la industria manufacturera, en espera de que al convertirse en un país altamente industrializado, lograría alcanzar mayor calidad de vida para los habitantes y podría competir con otros países altamente industrializados. Se pensó que la apertura económica que comenzó a principios de los años ochenta ameritaba incluir mayor valor agregado a sus exportaciones y en la especialización de éstas. Como consecuencia, logró destacar en la producción y exportación de productos electrónicos y maquinaria eléctrica, que se alimentaron de importaciones de distintos tipos de componentes; conductores, células fotoeléctricas, circuitos impresos, resistencias, entre otros (cuadro 4). 


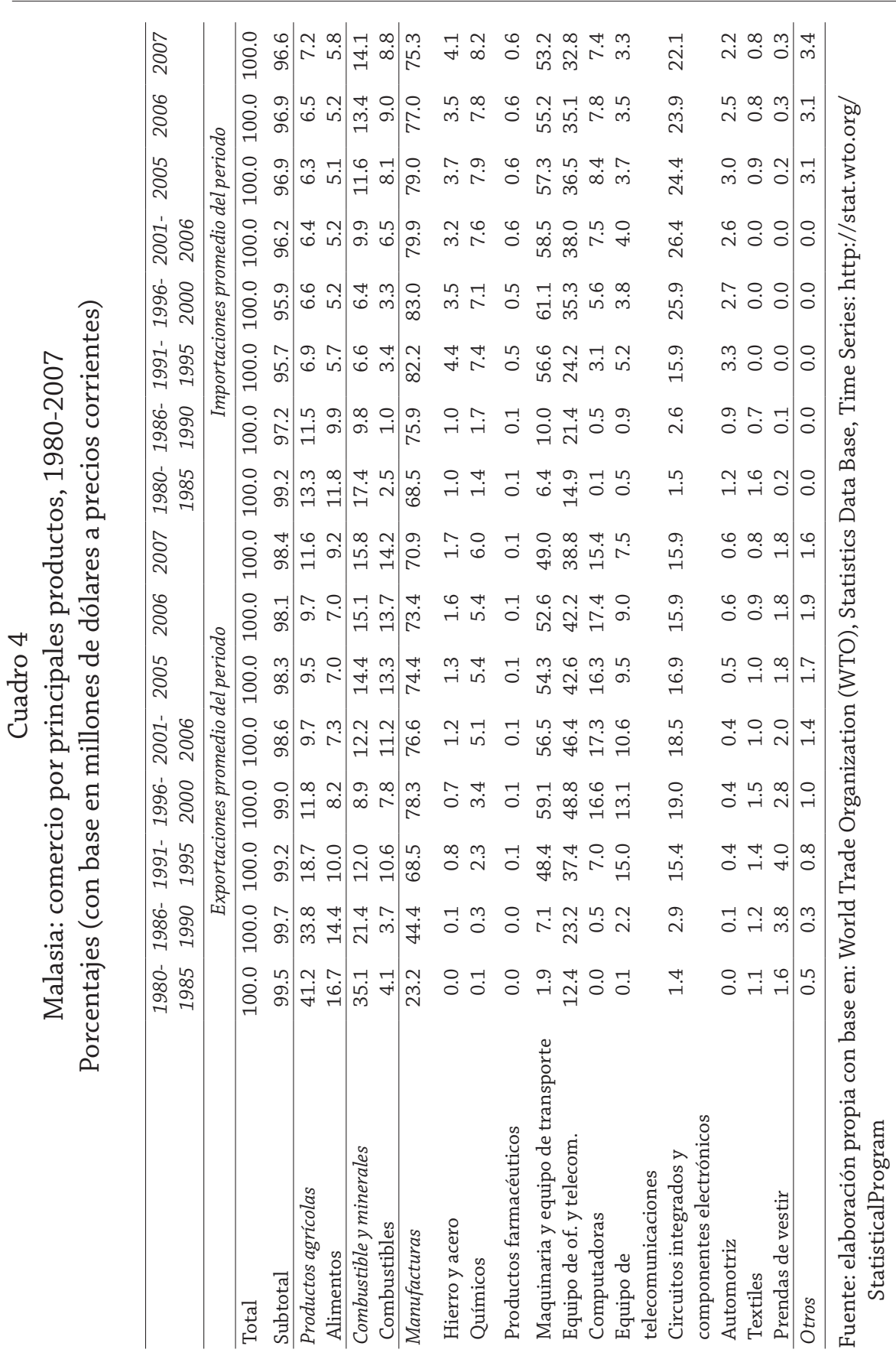


La composición del comercio hasta principios de los años ochenta se concentró en productos agrícolas, debido a la abundancia de aceite de palma, caucho natural, estaño, cacao, maderas tropicales, pimienta, reservas de petróleo y gas natural (compañía estatal de petróleo, Petronas).

Históricamente, Malasia ha sido importante en producciones agrícolas de caucho, cacao, pimienta, arroz, aceite de palma, además de estaño, petróleo y gas natural. En 1925 el caucho representó dos terceras partes del área cultivada en Malasia peninsular; a principios de los años noventa se mantenía como el principal abastecedor de caucho natural y en 1995 contribuyó con 51\% de la producción mundial de aceite de palma. ${ }^{10}$

Sin embargo, debido a la tendencia a la baja de los productos de origen agrícola y la falta de tecnología para industrializar los productos de origen vegetal y combustibles (gas y petróleo), el sector agrícola ha perdido protagonismo y la orientación de la política económica de Malasia ha sido buscar nuevos horizontes en producción que implique mayor valor agregado, y con ello atraer mayores ganancias.

La mayor parte de los intercambios de mercancías de Malasia es comercio de manufacturas (cuadros 2 y 4). En 2004 las exportaciones de manufacturas representaron $75 \%$ de las exportaciones totales de Malasia, porcentaje inferior al registrado en 2001: 80\%. Las máquinas de oficina y los equipos de telecomunicaciones siguieron ocupando el primer lugar entre las exportaciones en 2004, a pesar de que con 44.4\% descendieron respecto de 51\% de 2001.

La producción de equipos electrónicos depende en gran medida de partes y piezas importadas. Por la elevada dependencia respecto de los productos eléctricos y electrónicos, es mayor la vulnerabilidad ante las fluctuaciones de la demanda mundial de esos productos. La demanda mundial de bienes de tecnología de la información y las telecomunicaciones sacó a Malasia de la recesión en 1999; pero en 2001 el país sufrió las consecuencias de su descenso de la demanda. La estructura de las exportaciones sigue siendo relativamente igual que en 2001: los bienes exportados que reportan más ingresos son los circuitos electrónicos integrados, el aceite de palma y los productos basados en él, que se ha beneficiado de aumentos de los valores unitarios medios y de los volúmenes de exportación, las sustancias y productos químicos, el petróleo bruto y el gas natural licuado.

10. Martínez Oyarzabal María de Lourdes, Malasia, Centro de Estudios de Asia y África, El Colegio de México, 1997, p. 349. 


\section{Conclusiones}

Los acontecimientos económicos que llevaron al país al "milagro industrial de Malasia"11 se ha desvanecido poco a poco. La rápida transformación de las actividades económicas del país no dio tiempo de solucionar problemas estructurales que impiden, hasta la fecha, involucrar en la dinámica de crecimiento a todos los sectores de la economía.

El caso de Malasia muestra que no basta con lograr elevados porcentajes de crecimiento económico, sino que se tiene que evaluar el impacto de las políticas económicas en todos los sectores de la población. La migración del campo a la ciudad y la creciente brecha en la distribución del ingreso indican que hay mucho por hacer y que el gobierno debe corregir a tiempo las situaciones que impidan el crecimiento económico de forma incluyente y que ayuden a reducir la brecha entre los ricos y pobres.

La economía de Malasia es un claro ejemplo de que no es conveniente emprender el desarrollo económico con "brincos", en el aspecto tecnológico e industrial. Por el contrario, es necesario haber pasado por un proceso de formación de recursos humanos en forma ordenada y responder a los requerimientos acorde a sus políticas económicas, para lograr entender y asimilar los cambios en su economía.

La rectificación del gobierno en el Noveno Plan de Malasia representa la esperanza de encauzar el rumbo del país para lograr reducir la brecha en la disparidad del ingreso y con esto aminorar los problemas que surgieron por las decisiones tomadas en años anteriores. Seguramente no será sencillo el logro de los objetivos, debido a que los cambios estructurales en la economía tendrán que reorganizarse y reorientar la inversión a sectores estratégicos. Pero lo mejor de todo es que ya se ha marcado una nueva pauta en la historia económica de Malasia, sólo resta esperar los resultados para evaluar la efectividad de las políticas económicas que fueron incluidas en el noveno plan de Malasia. खֻ豸

11. Felker Grez y K. S. Jomo, op. cit., p. 2. 